\title{
Uniform Zariski's Theorem On Fundamental Groups
}

\author{
Shulim Kaliman \\ Department of Mathematics and Computer Science \\ University of Miami \\ Coral Gables, FL 33124
}

\section{Introduction.}

In [1] Zariski proved the following remarkable theorem. Let $\tilde{H}$ be an algebraic hypersurface in $\mathbb{C} \mathbb{P}^{n}$ where $n \geq 3$. Then for a generic projective plane $\tilde{A} \hookrightarrow \mathscr{C} \mathbb{P}^{n}$ the embedding $\tilde{A}-\tilde{H} \hookrightarrow \mathscr{C} \mathbb{P}^{n}-\tilde{H}$ generates an isomorphism $\pi_{1}(\tilde{A}-\tilde{H}) \rightarrow \pi_{1}\left(\mathbb{C} \mathbb{P}^{n}-\tilde{H}\right)$ of the fundamental groups. This implies the similar fact for an algebraic hypersurface $H$ in the Euclidean space $\mathbb{C}^{n}$. Consider a family of hypersurfaces $\tilde{H}_{p} \subset \mathbb{C} \mathbb{P}^{n}$ (resp. $H_{p} \subset \mathbb{C}^{n}$ ) depending algebraically on parameter $p$ from an algebraic variety $P$. It is natural to ask whether there exists a projective plane $\tilde{A}$ (resp. an affine plane $A$ ) such that the embedding $\tilde{A}-\tilde{H}_{p} \hookrightarrow \mathbb{C}^{\prime} \mathbb{P}^{n}-\tilde{H}_{p}$ (resp. $A-H_{p} \hookrightarrow \mathbb{C}^{n}-H_{p}$ ) generates an isomorphism of the corresponding fundamental groups for every $p$. In both cases the answer is negative, since it is enough to consider the set of all hyperplanes as this family of hypersurfaces depending algebraically on parameter. But the affine case has some advantage. Namely, we can change the coordinate system in $\mathbb{C}^{n}$ using a polynomial coordinate substitution (i.e., we can change the set of planes), whenever in the projective case we can use linear substitutions only. This observation leads to the main result of this paper.

For every family of algebraic hypersurfaces $H_{p}$ in $\mathbb{C}^{n}$ depending algebraically on parameter $p \in P$, one can choose a coordinate system in $\mathbb{C}^{n}$ in such a way that for some plane $A_{0} \subset \mathbb{C}^{n}$ the embedding $A_{0}-H_{p} \hookrightarrow \mathbb{C}^{n}-H_{p}$ generates an isomorphism $\pi_{1}\left(A-H_{p}\right) \rightarrow \pi_{1}\left(\mathbb{C}^{n}-H_{p}\right)$ for each $p$.

The scheme of the proof can be described as follows. The matter can be reduced to the three-dimensional case. Let $\rho: \mathbb{d}^{3} \rightarrow \mathbb{C}^{2}$ be a projection to an $(x, y)$-plane, let $A_{0}$ be $\rho^{-1}\left(L^{0}\right)$ where $L^{0}$ is the line $\{y=0\}$ in the $(x, y)$-plane. In section 3 we establish when the embedding $A_{0}-H \subset \mathbb{C}^{3}-H$ generates an isomorphism of the fundamental groups. It turns out that the following conditions are sufficient.

(1) The embedding $\rho^{-1}(o)-H \subset A_{0}-H$ generates an epimorphism of the fundamental groups where $o$ is the origin of the $(x, y)$-plane.

(2) The mapping $\left.\rho\right|_{H}: H \rightarrow \mathbb{C}^{2}$ is finite and, if it is $l$-sheeted, then the mapping $\left.\rho\right|_{H \cap A}: H \cap A \rightarrow L^{0}$ is also $l$-sheeted. 
(3) There exists a nonzero polynomial $g \in \mathbb{C}[x]$ such that $g(0)=0$ and for generic $c \in \mathbb{C}$ the number of points where the curve $L^{c}=\{y=c g(x)\}$ meets the image $\Gamma$ of the ramification set of the mapping $\left.\rho\right|_{H}$ coincides with the number of points where $L^{0}$ meets $\Gamma$.

The proof of this fact is a modification of the original argument of Zariski who dealt actually with the case when $g(x)=x$, i.e. $\left\{L^{c}\right\}$ is a pencil of lines.

The trouble with the linear coordinate substitutions is that those of them for which one of these three conditions does not hold form a subvariety of codimension 1 in the space of the linear coordinate substitutions. Therefore, for every family of hypersurfaces we shall construct a wider space of polynomial coordinate systems so that for every hypersurface from our family the subset of the coordinate systems in this space with one of these three conditions violated (relative to this hypersurface) has codimension at least $l$ where the "bigger" space of coordinate systems we choose the larger $l$ is. When $l$ is larger than the dimension of the parameter set $P$ we can find a coordinate system such that the embedding $A_{0}-H_{p} \subset \mathbb{C}^{3}-H_{p}$ generates an isomorphism of the fundamental groups for every $p$.

The paper is organized as follows. After preliminaries (section 2) we show that the embedding $A_{0}-H \hookrightarrow \mathbb{C}^{n}-H$ generates an isomorphism of the fundamental groups provided (1)-(3) hold. In section 4 we prove some technical fact which enable us to describe coordinate systems for which conditions (1)-(3) are true. In the last section we prove the main result for the three-dimensional case first and then we reduce the general case to the three-dimensional one.

\section{Terminology and Notation.}

In this paper $P$ and later $Q$ denote always sets of parameters, and a set of parameters is always an algebraic variety over the field of complex numbers.

Definition 2.1 Suppose that $T$ is a closed algebraic subvariety in $\mathbb{C}^{n} \times P$. Let $\rho_{1}: T \rightarrow \mathbb{C}^{n}$ and $\rho_{2}: T \rightarrow P$ be the natural projections and let $H_{p}=\rho_{1} \circ \rho_{2}^{-1}(p)$ where $p \in P$. Then we say that $\left\{H_{p}\right\}$ is a family of algebraic varieties with parameter $p \in P$. We shall mostly deal with the case when $H_{p}$ is a closed algebraic hypersurface in $\mathbb{C}^{n}$ for every $p \in P$. In this case we say that $\left\{H_{p}\right\}$ is a family of hypersurfaces in $\mathbb{C}^{n}$ with parameter $p \in P$. When $n=2$ we speak about a family of curves.

Remark 2.2 We do not suppose a priori that $P$ is smooth, irreducible, or that $P$ has a pure dimension. But we can always modify $P$ to make it simpler. For instance, we can treat $P$ as the disjoint union of the strata of its canonical stratification as a singular algebraic variety. Moreover, we can modify $P$ further. Consider, for instance, a function $\xi$ on $P$ with a finite number of values such that the preimage of each of these values is an algebraic subvariety of $P$. Consider a component $P^{\prime}$ of $P$ and 
suppose that $\xi$ is constant on $P^{\prime}-P_{0}^{\prime}$ where $P_{0}^{\prime}$ is a closed proper algebraic subvariety of $P^{\prime}$. We can replace $P^{\prime}$ by the disjoint union of $P^{\prime}-P_{0}^{\prime}$ and $P_{0}^{\prime}$. Then we can replace $P_{0}^{\prime}$ again by the disjoint union of the strata of its canonical stratification. Therefore, induction by dimension implies that one can suppose from the beginning that $P$ is smooth and $\xi$ is constant on every component of $P$. In particular, for every family of hypersurfaces $\left\{H_{p}\right\}$ we can suppose that the degree (or the Newton polygon) of the defining polynomial $f_{p}$ of $H_{p}$ is constant on every component of $P$. Each polynomial $f_{p}$ can be represented as $\prod_{i=1}^{l(p)}\left(f_{p, i}\right)^{n_{i}(p)}$ where $f_{p, i}$ are irreducible polynomials which are non-proportional for different values of $i$, and $n_{i}(p)$ are natural. We can suppose again that the function $l(p)$ is constant on every component of $P$.

Convention 2.3 Since we consider the fundamental group of the complement of a hypersurface it is natural to restrict ourselves to the case when this hypersurface is reduced. In the case of a family of hypersurfaces we can require that each generic hypersurface from this family is reduced. In general, even if a generic member of a family of hypersurfaces is reduced there may be some hypersurfaces which are not reduced. But dividing the set of parameters into a disjoint union of subsets in the manner described in Remark 2.2, we can suppose and we will suppose that every hypersurface in our family is reduced (for this we have to suppose that $l(p)$ from Remark 2.2 is constant on every component of $P$ and to replace $f_{p}=\prod_{i=1}^{l(p)}\left(f_{p, i}\right)^{n_{i}}$ by $\prod_{i=1}^{l(p)} f_{p, i}$ on each component of $P$ ).

Remark 2.4 We say that an algebraic variety $B$ depends algebraically on a polynomial $f$ if $B$ can be viewed as an member of a family of algebraic varieties whose parameter set is a Zariski open subset of the set of polynomials of fixed degree (or with a fixed Newton polygon).

Definition 2.5 Let $H$ be a reduced algebraic hypersurface in $\mathbb{C}^{n}$ and $A$ be a closed affine algebraic subvariety in $\mathbb{C}^{n}$. We say that $A$ is $H$ compatible if the embedding $i: A-H \hookrightarrow \mathbb{C}^{n}-H$ generates an epimorphism of the fundamental groups $i_{*}: \pi_{1}(A-$ $H) \rightarrow \pi_{1}\left(\mathbb{C}^{n}-H\right)$. We say that $A$ is strictly $H$ compatible if $i_{*}$ is an isomorphism. We say that $A$ is (strictly) compatible relative to a family of hypersurfaces if it is (strictly) $H$ compatible for every hypersurface $H$ in this family.

Definition 2.6 We say that some property holds for a generic point of an algebraic variety $P$ if for every irreducible component $P^{\prime}$ of $P$ there exists a proper closed algebraic subvariety $P_{0}^{\prime}$ such that this property is true for every $p \in P^{\prime}-P_{0}^{\prime}$.

In particular if we have several properties and each of them holds for a generic point of $P$, then all of them hold simultaneously for a generic point of $P$. 


\section{Modification of Zariski's approach for the non-linear case.}

In this section the projection $\rho: \mathbb{C}^{3} \rightarrow \mathbb{C}^{2}$ is given by $(x, y, z) \rightarrow(x, y)$ and $H \subset \mathbb{C}^{3}$ is a reduced hypersurface whose defining polynomial is $f$. Denote by $A_{0}$ the $(x, z)$-plane in $\mathbb{C}^{3}$ and by $L^{0}$ the $x$-axis in the $(x, y)$-plane (of course, $A_{0}=\rho^{-1}\left(L^{0}\right)$ ). Suppose that $g \in \mathbb{C}[x]$ is a nonzero polynomial such that $g(0)=0$.

Definition 3.1 We say that the $\mathscr{C}^{\prime}$-curve $L^{0}$ is appropriate with respect to the triple $(H, \rho, g)$ if the following properties hold

(1) The $z$-axis (i.e., the line $\rho^{-1}(o)$ where $o$ is the origin of the $(x, y)$-plane) is $H \cap A_{0}$ compatible in the plane $A_{0}$.

(2) The mapping $\left.\rho\right|_{H}: H \rightarrow \mathbb{C}^{2}$ is finite and, if it is $l$-sheeted, then the mapping $\left.\rho\right|_{H \cap A_{0}}: H \cap A_{0} \rightarrow L^{0}$ is also $l$-sheeted.

(3) For generic $c \in \mathbb{C}^{r}$ the number of points where the curve $L^{c}:=\{y=c g(x)\}$ meets the image $\Gamma$ of the ramification set of the mapping $\left.\rho\right|_{H}$ coincides with the number of points where $\Gamma$ meets $L^{0}$.

The aim of this section is

Theorem 3.2 Let $L^{0}$ be appropriate with respect to $(H, \rho, g)$. Then $A_{0}$ is strictly H compatible.

In the case when $g$ is linear this theorem can be extracted from the original paper of Zariski [1]. In general case we follow also the outline of his arguments. The proof consists of several lemmas and we discuss first some additional notation which will be used further in these lemmas.

Let $X$ be the set of points where $L^{0}$ meets $\{g(x)=0\}$. The family $\left\{L^{c}\right\}$ can be viewed as a linear system of curves whose base point set is $X$. This linear system of curves generates the mapping $\tau: \mathbb{C}^{2}-X \rightarrow \mathbb{C} \mathbb{P}^{1}$ such that $\tau^{-1}(c)=L^{c}-X$ for $c \in \mathbb{C}^{1} \mathbb{P}^{1}$ (where $L^{\infty}=\{g(x)=0\}$ ). There exists a finite set $C=\left\{c_{1}, \ldots, c_{r}\right\} \subset \mathbb{C}^{\prime}$ such that for every $c \in \mathbb{C}-C$ the curve $\tau^{-1}(c)$ meets $\Gamma$ at the same number of points.

Put $Z=X \cup \tau^{-1}(C \cup \infty)$ (i.e. $\left.Z=L^{c_{1}} \cup \ldots \cup L^{c_{r}} \cup L^{\infty}\right)$. Set $\Gamma^{\prime}=\Gamma-Z$ (i.e. $\left.\Gamma^{\prime}=\Gamma \cap \tau^{-1}\left(C^{\prime}-C\right)\right)$ and $\Gamma^{\prime \prime}=\rho^{-1}\left(\Gamma^{\prime}\right) \cap H$. The set of points where a generic curve $L^{c}$ meets $\Gamma$ non-normally is contained in $X$, by condition (3). Hence every non-smooth point of $\Gamma$ is contained in $Z$ since at these points none of generic $C^{\prime}$-curves $L^{c}$ can meet $\Gamma$ normally. Hence $\Gamma^{\prime}$ is smooth and we need to show that the mapping $\left.\rho\right|_{\Gamma^{\prime \prime}}: \Gamma^{\prime \prime} \rightarrow \Gamma^{\prime}$ is unramified. This fact can checked locally.

Lemma 3.3 Let $\mathcal{H}$ be the germ of an analytic surface at the origin of $\mathbb{C}^{3}$. Suppose that the mapping $\rho_{0}: \mathcal{H} \rightarrow\left(\mathbb{C}^{2}, o\right)$ is finite where $\rho_{0}=\left.\rho\right|_{\mathcal{H}}$. Let $\gamma \subset\left(\mathbb{C}^{2}, o\right)$ be the image of the ramification set for the mapping $\rho_{0}$ and let $\mathcal{K}=\rho_{0}^{-1}(\gamma)$. Suppose that $\gamma$ 
is smooth and $\rho_{\mathcal{K}}: \mathcal{K} \rightarrow \gamma$ is the restriction of $\rho$ to $\mathcal{K}$. Then $\rho_{\mathcal{K}}$ is unramified.

Proof. Let $\mathcal{L}$ be the preimage of a generic point $b \in\left(\mathbb{C}^{2}, o\right)-\gamma$ under the mapping $\rho_{0}$. The fundamental group of $\left(\mathbb{C}^{2}, o\right)-\gamma$ is isomorphic to the group of integers since $\gamma$ is smooth and this group acts on $\mathcal{L}$. Hence $\mathcal{L}$ can be represented as the disjoint union of minimal invariant subsets of $\mathcal{L}$ relative to this action. These subsets correspond to the irreducible components of $\mathcal{H}$. Let $s$ be the number of points in the preimage $\mathcal{L}_{0}$ of a generic point $a \in \gamma$ under $\rho_{0}$. Sending $b$ to $a$ one can see that the points of $\mathcal{L}_{0}$ generate a partition of $\mathcal{L}$ into disjoint subsets. Using the fact that the fundamental group of $\left(\mathbb{C}^{2}, o\right)-\gamma$ is the group of integers, one can check that this partition is invariant under the action of the fundamental group (it is enough to consider the action on $\mathcal{L}$ of a small simple loop from $\left(\mathbb{C}^{2}, o\right)-\gamma$ around $a$ since this loop can be viewed as a generator of the fundamental group). Hence we can represent $\mathcal{H}$ as the union $\bigcup_{i=1}^{s} \mathcal{H}_{i}$ of the germs of surfaces so that $\mathcal{H}_{i} \cap \rho^{-1}(\gamma)=\mathcal{K}_{i}$ where $\mathcal{K}_{i} \subset \mathcal{K}$ are the germs of different curves with the origin as the only common point (since $\mathcal{K}_{i} \cap \rho^{-1}(a)$ is exactly one point in $\mathcal{L}_{0}$ that corresponds to $\mathcal{H}_{i}$ ). Assume that $s \geq 2$. Consider the germ of the curve $\zeta=\mathcal{H}_{1} \cap \mathcal{H}_{2}$. By construction, the germ $\rho(\zeta)$ meets $\gamma$ at the origin only. But it must be contained in $\gamma$ since $\zeta$ is contained in the ramification set. Contradiction. Hence $s=1$ which is the desired conclusion. QED.

Put $Y=\tau^{-1}(\mathbb{C}-C) \cup X$. For every subset $K$ of $\mathbb{C}^{2}$ we denote by $\tilde{K}$ the set $\rho^{-1}(K)-H$. For instance, $\tilde{Y}=\rho^{-1}(Y)-H$.

Lemma 3.4 The natural embedding $i: \tilde{Y} \hookrightarrow \mathbb{C}^{3}-H$ generates an isomorphism of the fundamental groups $i_{*}: \pi_{1}(\tilde{Y}) \rightarrow \pi_{1}\left(\mathbb{C}^{3}-H\right)$.

Proof. By condition (3) in Definition 3.1, $C$ does not contain 0. Since $\tau^{-1}(0)=$ $L^{0}-X$ the set $\tilde{Y}$ contains $A_{0}-H$. We shall see later (Lemma 4.2) that under condition (2) every line which is parallel to the $z$-axis and which meets $H$ at $l$ points, is $H$ compatible. Thus condition (2) implies that $A_{0}$ is $H$ compatible. Hence $i_{*}$ is an epimorphism.

Let $\delta_{1}, \ldots, \delta_{s}$ be a set of generators in $\pi_{1}(\tilde{Y})$, and, therefore, it can be treated as a set of generators in $\pi_{1}\left(\mathbb{C}^{3}-H\right)$. We need to show that if $\delta_{1}, \ldots, \delta_{s}$ satisfy some generating relations in $\pi_{1}\left(\mathbb{C}^{3}-H\right)$ then they satisfy the same relations in $\pi_{1}(\tilde{Y})$. For this purpose it suffices to show that for every 2-cell $\Delta_{1}$ in $\mathbb{C}^{3}-H$ with a boundary $\partial \Delta_{1} \subset \tilde{Y}$ there exists a 2-cell $\Delta_{2} \subset \tilde{Y}$ with the same boundary. We can suppose that if $u$ is an intersection point of $\tilde{Z}$ and the interior of $\Delta_{1}$ then $\Delta_{1}$ meets $\tilde{Z}$ normally at $u$, and $u \notin \tilde{X}$. (We can do this since the real codimension of $\tilde{X}$ in $\mathbb{C}^{3}-H$ is 4.) Since $X$ is the base point set for $\left\{L^{c}\right\}$ one can choose a path $\xi$ in $\tilde{Z}$ joining $u$ and a point $v \in \tilde{X}$ in such manner that $\rho(v)$ is the only point from $\rho(\xi)$ that belongs to $X$ and that $\xi-v$ is contained in the smooth part $\tilde{Z}^{*}$ of $\tilde{Z}$. One can identify a neighborhood of $\tilde{Z}^{*}$ 
in $\mathbb{C}^{3}$ with a neighborhood of the zero section of the normal bundle to $\tilde{Z}^{*}$. Moreover, we can suppose that the intersection of $\Delta_{1}$ with this neighborhood is contained in a fiber of this bundle. Choose a small 2-cell $\Delta_{\varepsilon}(u) \subset \Delta_{1}$ with center at $u$ and replace it by a cone in $\tilde{Y}$ with the following properties: $v$ is the vertex of the cone and the only point where the cone meets $\tilde{Z}$, the base of the cone coincides with the boundary of $\Delta_{\varepsilon}(u)$, the intersection of the cone with the fiber of the normal bundle to $\tilde{Z}^{*}$ at every point of $\xi-v$ is a circle. Repeating this procedure we obtain a 2-cell $\Delta_{2} \subset \mathbb{C}^{3}-H$ such that its boundary coincides with the boundary of $\Delta_{1}$ and its interior meets $\tilde{Z}$ only at points from $\tilde{X}$. In particular, $\Delta_{2} \subset \tilde{Y}$.

QED.

Let $\sigma_{1}, \ldots, \sigma_{r}$ be a bouquet of simple loops in $\mathbb{C}-C$ with one common point at the origin so that these loops generate the fundamental group of $\mathscr{C}-C$. Put $B=\tau^{-1}\left(\bigcup_{k} \sigma_{k}\right) \cup X$. We have to show that $\tilde{B}$ is a deformation retract of $\tilde{Y}$, and, in particular, the embedding $j: \tilde{B} \hookrightarrow \tilde{Y}$ generates an isomorphism $j_{*}: \pi_{1}(\tilde{B}) \rightarrow \pi_{1}(\tilde{Y})$. The construction of this deformation can be reduced to a simpler problem due to

Lemma 3.5 Let $K$ be a subset of $Y$. Suppose that $\kappa=\left\{\kappa_{t} \mid t \in[0,1]\right\}$ is a path in the space of continuous mappings from $K$ to $Y$ such that $\kappa_{0}$ is the identical embedding, the restriction of $\kappa_{t}$ to $K \cap X$ is the identical embedding for every $t \in[0,1]$, $\kappa_{t}(\Gamma \cap K) \subset \Gamma$, and $\kappa_{t}(K-(X \cup \Gamma)) \subset Y-(X \cup \Gamma)$. Suppose also that the restriction of $\kappa$ to $(K-X) \times[0,1]$ is smooth. Then there exists a deformation $D=\left\{D_{t} \mid t \in[0,1]\right\}$ of the identical embedding $D_{0}: \tilde{K} \hookrightarrow \tilde{Y}$ such that $\rho D=\kappa \rho$ and $D$ is identical on $\tilde{K} \cap \tilde{X}$. Moreover, if for every $t$ the mapping $\kappa_{t}$ is a homeomorphism between $K$ and $\kappa_{t}(K)$ then $D_{t}$ is a homeomorphism between $\tilde{K}$ and $D_{t}(\tilde{K})$.

Proof. Fix a neighborhood $U$ of $H-\rho^{-1}(X)$ in $\mathbb{C}^{3}$ so that $\bar{U} \cap \rho^{-1}(b)$ is compact for every $b \in \mathbb{C}^{2}\left(\bar{U}\right.$ is, of course, the closure of $U$ in $\left.\mathbb{C}^{3}\right)$. Suppose also that $\bar{U} \cap \rho^{-1}(X)=$ $H \cap \rho^{-1}(X)$, i.e this set is finite. Consider the natural projection $T \mathbb{C}^{2} \rightarrow \mathbb{C}^{2}$ where $T \mathbb{C}^{2}$ is the tangent bundle of $\mathscr{C}^{2}$, and the mapping $\rho: \mathbb{C}^{3} \rightarrow \mathbb{C}^{2}$. They generate the set $T=\mathbb{C}^{3} \otimes_{\mathbb{C}^{2}} T \mathbb{C}^{2}$ with the natural projections $p r_{1}: T \rightarrow \mathbb{C}^{3}$ and $p r_{2}: T \rightarrow T \mathbb{C}^{2}$. Put $Y^{\prime}=\mathbb{C}^{3}-\left(H \cap \rho^{-1}(X \cup \Gamma)\right)$ and put $W=\operatorname{pr}_{1}^{-1}\left(Y^{\prime}\right)$. Let $W_{0}=\operatorname{pr}_{1}^{-1}\left(\tilde{\Gamma}^{\prime}\right) \cap p r_{2}^{-1}\left(T \Gamma^{\prime}\right)$ and $\bar{W}_{0}=p r_{1}^{-1}\left(\rho^{-1}\left(\Gamma^{\prime}\right)\right) \cap p r_{2}^{-1}\left(T \Gamma^{\prime}\right)$ where $T \Gamma^{\prime}$ is the tangent bundle to $\Gamma^{\prime}$. Using partition of unity one can construct a smooth mapping $\chi: W \rightarrow T Y^{\prime}$ where $T Y^{\prime}$ is the tangent bundle of $Y^{\prime}$ with the following properties:

(i) for every $w \in W$ we have $\rho_{*} \chi=p r_{2}$;

(ii) for every $w \in W$ with $p r_{1}(w) \notin U$ the $z$-coordinate of the vector $\chi(w)$ is zero;

(iii) for every $w \in W$ with $p_{1}(w) \in H$ the vector $\chi(w)$ is tangent to $H$. (Note that this tangent vector exists since the restriction of $\rho$ to $H-\rho^{-1}(\Gamma)$ is an unramified covering of $\mathbb{C}^{2}-\Gamma$ );

(iv) the restriction of $\chi$ to $W_{0}$ can be extended to a smooth mapping $\chi_{0}: \bar{W}_{0} \rightarrow$ 
$T \mathbb{C}^{3}$ (where $T \mathbb{C}^{3}$ is the tangent bundle of $\mathbb{C}^{3}$ ) so that for every $w \in \bar{W}_{0}$ with $p r_{1}(w) \in$ $\Gamma^{\prime \prime}$ the vector $\chi_{0}(w)$ is tangent to $\Gamma^{\prime \prime}$. (This vector $\chi_{0}(w)$ exists since the restriction of $\rho$ to $\Gamma^{\prime \prime}$ is an unramified covering of $\Gamma^{\prime}$, by Lemma 3.3.)

It is worth mentioning that we need condition (iv) separately from (iii) since in general the mapping $\chi$ cannot be extended continuously to the points $w$ with $\operatorname{pr}_{1}(w) \in \rho^{-1}(\Gamma) \cap H$.

Consider the curve $\kappa(b)=\left\{\kappa_{t}(b) \mid t \in[0,1]\right\}$ for $b \in K-X$. Suppose for simplicity that it has no selfintersection points (otherwise we can replace the curve with its graph). Then $\widetilde{\kappa(b)}$ is a smooth real manifold. For each $u_{0} \in \widetilde{\kappa(b)}$ there exists $t_{0} \in[0,1]$ such that $\rho\left(u_{0}\right)=\kappa_{t_{0}}(b)$. Let $v_{b}\left(t_{0}\right)$ be the vector tangent to $\kappa(b)$ at $\kappa_{t_{0}}(b)$ which is generated by differentiation with respect to $t$. Then the vector $\chi\left(u_{0} \otimes v_{b}\left(t_{0}\right)\right)$ is tangent to $\widetilde{\kappa(b)}$ at $u_{0}$. Therefore, such vectors define a vector field on $\widetilde{\kappa(b)}$. Let $\rho(u)=b$ and let $D(u)=\left\{D_{t}(u) \mid t \in[0,1]\right\}$ be the integral curve of this vector field such that it begins at the point $u=D_{0}(u)$. These curves define a deformation $\mathrm{D}$ of $\tilde{K}-\tilde{X}$ in $\tilde{Y}-\tilde{X}$ with $\rho D=\kappa \rho$ unless for some $u \in \tilde{K}-\tilde{X}$ the curve $D(u)$ goes either to infinity or to $H$ for a finite time. It cannot go infinity for a finite time within $U$ due to the fact that $\bar{U} \cap \rho^{-1}(b)$ is compact for every $b$ and the mapping $\left.\rho\right|_{H}$ is finite. Outside $U$ it cannot go to infinity as well, by (ii). When $b \notin \Gamma$ the curve $D(u)$ cannot reach $H$ for a finite time due to (iii). When $b \in \Gamma$ the curve $\kappa(b) \subset \Gamma$, by the assumption of Lemma, and $D(u)$ cannot reach $H$ again for a finite time due to (iv). Therefore, $D(u) \subset \tilde{Y}$ and $D_{t}(\tilde{K}-\tilde{X}) \subset \tilde{Y}$ for every $t$.

Note that the combination of (ii) and the facts that $\left.\kappa_{t}\right|_{K \cap X}$ is the identical embedding and that the set $\bar{U} \cap \rho^{-1}(X)$ is finite implies that $D$ can be extended to $\tilde{X}$ by the identical deformation which proves the first statement of Lemma. The second statement follows obviously from the construction of $D$. QED.

The next lemma is almost the exact repetition of Lemma 3.5 but we give its proof for the sake of completeness.

Lemma 3.6 Let $K \subset Y$ and $\kappa_{0}: K \rightarrow Y$ be the identical embedding. Let $\theta_{0}$ be the identical embedding of $\tau(K-X)$ into $\mathscr{C}-C$. Suppose that there exists a smooth path $\theta=\left\{\theta_{t} \mid t \in[0,1]\right\}$ in the space of smooth mappings from $\tau(K-X)$ to $\mathcal{C}-C$. Then there exist a deformation $\kappa=\left\{\kappa_{t}\right\}$ of $\kappa_{0}$ so that $\left.\kappa_{t}\right|_{X}$ is the identical embedding for every $t, \kappa(\Gamma \cap K) \subset \Gamma, \kappa(K-(X \cup \Gamma)) \subset Y-(X \cup \Gamma)$, and $\left.\tau \circ \kappa\right|_{K-X}=\left.\theta \circ \tau\right|_{K-X}$. Moreover, if for every $t$ the mapping $\theta_{t}$ is a diffeomorphism between $\tau(K-X)$ and its image then $\kappa_{t}$ is a homeomorphism between $K$ and its image, and the restriction of $\kappa$ to $(K-X) \times[0,1]$ is smooth.

Proof. Note that $Y-X=\tau^{-1}(\mathbb{C}-C)$ can be treated as $\left(\mathbb{C}-\left\{x_{1}^{0}, \ldots, x_{l}^{0}\right\}\right) \times(\mathbb{C}-$ 
$C) \subset \mathbb{C} \times(\mathbb{C}-C)$ where $x_{1}^{0}, \ldots, x_{l}^{0}$ are the $x$-coordinates of the points of $X$. Then $\tau$ can be treated as the natural projection $\tau_{2}$ to the second factor. Let $\tau_{1}: \tau^{-1}\left(\mathbb{C}^{\prime}-C\right) \rightarrow \mathscr{C}$ be the projection to the first factor. Choose a small tubular neighborhood $U$ of $\Gamma^{\prime}$ in $\tau^{-1}(\mathbb{C}-C)$ such that its closure $\bar{U}$ in $\mathscr{C} \times\left(\mathscr{C}^{\prime}-C\right)$ does not meet the sets $x_{i}^{0} \times(\mathbb{C}-C), i=1, \ldots, l$. Using partition of unity we can construct a vector field $\mu$ on $\mathbb{C} \times(\mathbb{C}-C)$ so that

- outside $U$ we have $\tau_{1 *}(\mu) \equiv 0$,

- $\mu$ is tangent to $\Gamma^{\prime}$

- $\tau_{2 *}(\mu)$ is a nonzero constant vector field on $\mathbb{C}^{\prime}-C$ (this means that one can suppose that the phase flow associated with $\mu$ transforms $L^{c}$ into $L^{c+t}$ for time $t$ whenever this flow is defined correctly).

If $b \in K \cap X$ put $\kappa(b)=b$ and if $b \in K-X$ define $\kappa_{t}(b)$ as follows. Consider $c=\tau(b)$ and $M_{c}=\tau^{-1}(\theta(c))$. Suppose for simplicity that $\theta(c)$ has no selfintersection points. Then $M_{c}$ is a smooth real manifold which is naturally embedded in $\tau^{-1}(\mathbb{C}-C)$. For each $a \in M_{c}$ there exists $t$ such that $\tau(a)=\theta_{t}(c)$. Consider the vector $\theta_{t}^{\prime}(c) \mu(a)$ at $a$ where $\theta_{t}^{\prime}(c)$ is the derivative of the function $\theta(c):[0,1] \rightarrow \mathbb{C}$ with respect to $t$. This vector is tangent to $M_{c}$ and, therefore, such vectors define a vector field $\mu_{c}$ on $M_{c}$. This vector field defines uniquely an integral curve $\kappa_{t}(b)$ in $M_{c}$ which begins at $b=\kappa_{0}(b)$.

The continuity of $\kappa$ is clear unless for some $b \in K-X$ the curve $\kappa(b)$ goes either to infinity or to $x_{i}^{0} \times(\mathbb{C}-C)$ for a finite time. But it cannot go to infinity since we cannot reach infinity within $U$ for a finite time due to the description of $U$, and outside $U$ the behavior of $\kappa_{t}(b)$ is defined by the vector field $\mu$ which does not send points from $\tau^{-1}\left(C^{\prime}-C\right)$ to infinity since $\tau_{1 *}(\mu)=0$. Similarly, a set $\left.x_{i}^{0} \times(\mathscr{C}-C)\right)$ cannot be reached for a finite time. Thus $\kappa$ is continuous and $\kappa_{t}(K-X) \subset Y-X$ for every $t$. Note that $\mu_{c}$ is tangent to $\Gamma \cap M_{c}$. Hence the curve $\kappa(b)$ is either contained in $\Gamma$ or does not meet it which yields the desired properties of $\kappa$ in the first statement. The second statement follows obviously from the construction of $\kappa$ and the fact that the integral curve $\kappa(b)$ depends smoothly on $b \in K-X$.

QED.

The Proof of Theorem 3.2. We can suppose that the bouquet $\bigcup_{k} \sigma_{k}$ is chosen so that there exists a smooth deformation $\theta$ of $\mathbb{C}-C$ to this bouquet. By Lemma 3.6, there exists a deformation $\kappa=\left\{\kappa_{t}\right\}$ of $Y$ to $B$ with the prescribed properties. By Lemma 3.5, there exists a deformation of $\tilde{Y}$ to $\tilde{B}$ which is identical on $\tilde{X}$. Thus it remains to show that the natural embedding of $A_{0}-H$ into $\tilde{B}$ generates an isomorphism of the fundamental groups. As we mentioned in the proof of Lemma 3.4 this embedding generates an epimorphism of the fundamental groups. By (1) the line $\rho^{-1}(o)$ is $H$ compatible. Hence the loops $\delta_{1}, \ldots, \delta_{s}$ which generate $\pi_{1}\left(\rho^{-1}(o)-H\right)$ can be viewed as generators of $\pi_{1}(\tilde{B})$. If we travel along a loop $\sigma_{j}:[0,1] \rightarrow \mathbb{C}-C$ from the 
point $o=\sigma_{j}(0)$ to a point $c=\sigma_{j}(t)$ then Lemma 3.6 provides us with an appropriate homeomorphism between $L^{0}$ and $L^{c}$ which depends continuously on $t$. It generates in turn a homeomorphism (depending continuously on $t$ ) between $\tilde{L}^{0}\left(=A_{0}-H\right.$ ) and $\tilde{L}^{c}$ which is identical on $\tilde{X}=\tilde{L}^{0} \cap \tilde{L}^{c}$, by Lemma 3.5. Thus after traveling along the whole loop $\sigma_{j}$ we deform each element $\delta \in \pi_{1}\left(\tilde{L}^{0}\right)$ into another element $\delta^{(j)} \in \pi_{1}\left(\tilde{L}^{0}\right)$. By the van Kampen theorem [3], the generating relations for $\delta_{1}, \ldots, \delta_{s}$ in $\pi_{1}\left(\tilde{L}^{0}\right)$ together with the relations $\delta_{i}=\delta_{i}^{(j)}$ give all the generating relations between $\delta_{1}, \ldots, \delta_{s}$ in $\pi_{1}(\tilde{B})$. But since for every $j$ the homeomorphism of $\tilde{L}^{0}$ generated by the loop $\sigma_{j}$ is identical on $\tilde{X}$ the loops $\delta_{i}$ and $\delta_{i}^{(j)}$ coincide for every $i$. Hence the embedding of $\rho^{-1}(o)-H$ into $\tilde{B}$ generates an isomorphism of the fundamental groups. Since $\tilde{B}$ is a deformation retract of $\tilde{Y}$, Lemma 3.4 implies that the embedding of $A_{0}-H$ into $\mathbb{C}^{3}-H$ generates also an isomorphism of the fundamental groups.

QED.

\section{Technical Facts}

In this section we shall describe polynomial coordinate substitutions which lead eventually to conditions (1)-(3) from Definition 3.1. The fist two lemmas of this section are formulated for hypersurfaces in $\mathbb{C}^{3}$ but they can be easily reformulated for hypersurfaces in $\mathbb{C}^{n}$ with $n \geq 3$.

Lemma 4.1 Let $\left(x_{1}, y_{1}, z_{1}\right)$ be a coordinate system in $\mathbb{C}^{3}$ and let $\left\{H_{p} \subset \mathbb{C}^{3} \mid p \in\right.$ $P\}$ be a family of hypersurfaces with defining polynomials $\left\{f_{p}\right\}$. Suppose that $d$ is natural such that $d>\max _{p \in P} \operatorname{deg} f_{p}$. Let $(x, y, z)$ be a new coordinate system such that $\left(x_{1}, y_{1}, z_{1}\right)=\left(g_{1}(x, y, z), g_{2}(x, y, z), z\right)$ where $g_{1}$ and $g_{2}$ are polynomials. Suppose that the degrees of $g_{1}(a, b, z)$ and $g_{2}(a, b, z)$ are $d_{1}$ and $d_{2}$ respectively for every constants a and $b$. Let $d_{2}>d$ and $d_{1}>d \cdot d_{2}$. Then the restriction of the projection $\rho(x, y, z) \rightarrow$ $(x, y)$ to every hypersurface $H_{p}$ is a finite morphism.

Proof. Fix $p \in P$. The restriction of $\rho$ to $H_{p}$ is finite if for every constants $a$ and $b$ the degree of the polynomial $\varphi(z)=f_{p}\left(g_{1}(z, a, b), g_{2}(z, a, b), z\right)$ does not depend on $a$ and $b$. Consider monomials $x_{1}^{k} y_{1}^{l} z_{1}^{m}$ which are present in $f_{p}$ with nonzero coefficients and consider the vectors $(k, l, m)$. Suppose that $\left(k^{0}, l^{0}, m^{0}\right)$ is the greatest among these vectors in the lexicographic order. One can see that $\operatorname{deg} \varphi$ coincides then with $k^{0} d_{1}+l^{0} d_{2}+m$ regardless of the choice of $a$ and $b$.

QED.

Lemma 4.2 Let $\rho: \mathbb{C}^{3} \rightarrow \mathbb{C}^{2}$ be the projection given by $(x, y, z) \rightarrow(x, y)$. Suppose that a reduced hypersurface $H$ in $\mathbb{C}^{3}$ does not contain lines parallel to the z-axis. Consider a line $L=\rho^{-1}(w)$ with $w \in \mathbb{C}^{2}$ which meets $H$ at $\operatorname{deg}_{z} f$ points (counting without multiplicity). Then $L$ is $H$ compatible. 
Proof. There is an algebraic subvariety $S \subset \mathbb{C}^{2}$ such that the line $\rho^{-1}(s)$ meets $H$ at less than $\operatorname{deg}_{z} f$ points counting without multiplicity iff $s \in S$. Put $E=$ $\mathbb{C}^{3}-\left(H \cup \rho^{-1}(S)\right)$ and $\tau=\left.\rho\right|_{E}$. Then the mapping $\tau: E \rightarrow \mathbb{C}^{2}-S$ is a fibration whose generic fiber $F$ is a $\operatorname{deg}_{z} f$ times punctured complex line. Let $i: F \rightarrow E$ be the natural embedding. We have the exact sequence of the fundamental groups

$$
\rightarrow \pi_{1}(F) \stackrel{i_{*}}{\rightarrow} \pi_{1}(E) \stackrel{\tau_{*}}{\rightarrow} \pi_{1}\left(\mathbb{C}^{2}-S\right) \rightarrow 0 .
$$

Choose simple loops $\left\{\sigma_{i}\right\}$ in $\mathbb{C}^{2}-S$ around each point in a finite set $\left\{s_{i}\right\} \subset S$ such that these loops generate the whole fundamental group $\pi_{1}\left(\mathbb{C}^{2}-S\right)$. Since $\rho^{-1}\left(s_{i}\right)$ is not contained in $H$ one can choose a loop $\gamma_{i}$ in $E$ so that it is contractible in $\mathbb{C}^{3}-H$ and $\tau\left(\gamma_{i}\right)=\sigma_{i}$. Then the exact sequence implies that every element in $\pi_{1}(E)$ can be written in the form $u v$ where $v \in i_{*}\left(\pi_{1}(F)\right)$ and $u$ lies in the group generated by $\left\{\left[\gamma_{i}\right]\right\}$. Consider the embedding $j: E \rightarrow \mathbb{C}^{3}-H$ and the corresponding homomorphism of the fundamental groups $j_{*}: \pi_{1}(E) \rightarrow \pi_{1}\left(\mathbb{C}^{2}-H\right)$. Note that $\left[\gamma_{i}\right] \in \operatorname{ker} j_{*}$ and $j_{*}(u v)=j_{*}(v)$. On the other hand $j_{*}$ is surjective, of course. Hence $j_{*} \circ i_{*}\left(\pi_{1}(F)\right)=\pi_{1}\left(\mathbb{C}^{3}-H\right)$ and we are done, since $L-H$ is a generic fiber of $\tau$. QED.

We shall cite notation which will be used in the remainder of this section. A curve $\Gamma$ in $\mathbb{C}^{2}$ is always reduced and it coincides with the zero locus of a polynomial $f$. We shall denote by $h^{0}$ a polynomial in one variable of degree $d_{0}$. We shall consider $\mathbb{C}_{\text {-curves in }} \mathbb{C}^{2}$ given by equations of form $y+h^{0}(x)+g(x)=0$ where $g$ runs over the space of polynomials $G_{m}$ of degree $m<d_{0}$. The family of these curves will be denoted by $V_{m}\left(h^{0}\right)$. There is a natural bijection between $G_{m}$ and $V_{m}\left(h^{0}\right)$ and we denote by $L_{g}$ the $\mathcal{C}_{\text {-curve from }} V_{m}\left(h^{0}\right)$ that corresponds to $g \in G_{m}$.

Lemma 4.3 Let $\Gamma$ be an algebraic curve in $\mathbb{C}^{2}$ which does not contain lines parallel to the $y$-axis. Let a polynomial $g$ has a simple root $x^{0}$ so that the line $C=\left\{x=x^{0}\right\}$ meets $\Gamma$ at $\operatorname{deg}_{y} f$ different points. Let $g^{0} \in G_{m}$ and $g(c)=g^{0}+c g$. Then the curve $L_{g(c)} \in V_{m}\left(h^{0}\right)$ is $\Gamma$ compatible when $|c|$ is sufficiently large.

Proof. By Lemma 4.2, $C$ is $\Gamma$ compatible. When $|c| \rightarrow \infty$ then $L_{g(c)}$ approaches to $C$. Repeating the argument of [2, Lemma 3], one can see that $L_{g(c)}$ is $\Gamma$ compatible for large $|c|$. (In $[2$, Lemma 3] we used the term " $\Gamma$ proper" instead of " $\Gamma$ compatible". We made this replacement since the term "proper" may be misunderstood.) QED.

Remark 4.4 Suppose that $\omega \in G_{m}$ is a nonzero polynomial and $g^{0} \in G_{m}$. Let

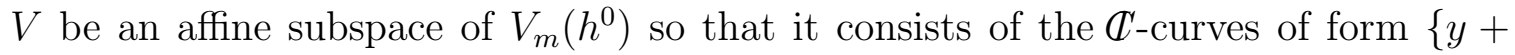
$\left.h^{0}(x)+g^{0}(x)+\omega(x) \tilde{g}(x)=0\right\}$ where $\tilde{g} \in G_{n}$ and $n=m-\operatorname{deg} \omega$. Note that if $n>0$ then for generic $\tilde{g} \in G_{n}$ the polynomial $g=\tilde{g} \omega$ has always a simple root $x^{0}$ such that the line $C$ described in Lemma 4.3 is $\Gamma$ compatible. 
Lemma 4.5 Suppose that the curve $L_{g^{0}}$ meets $\Gamma$ at the same number of points as

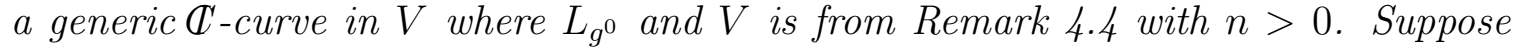
also that $\Gamma$ does not contain lines parallel to the $y$-axis. Then $L_{g^{0}}$ is $\Gamma$ compatible.

Proof. Put $\mathcal{L}=\left\{((x, y), L) \in \mathbb{C}^{2} \times V \mid(x, y) \in L-\Gamma\right\}$. Let $\kappa: \mathcal{L} \rightarrow V$ be the natural projection. Then there exists a closed algebraic subvariety $S$ of $V$ such that a curve $L$ from $V$ meets $\Gamma$ at a less number of points than the generic curve from $V$ iff this curve $L$ is from $S$. Hence the restriction of $\kappa$ to $\kappa^{-1}(V-S)$ is a fibration over $V-S$. This fibration provides an isotopy between $L_{g^{0}}-\Gamma$ and $L-\Gamma$ in $\mathbb{C}^{2}-\Gamma$ where $L$ is a generic curve in $V$. By Lemma 4.3 and Remark 4.4, $L$ and, therefore, $L_{g^{0}}$ are $\Gamma$ compatible.

QED.

The number of points at which $L_{g}$ meets $\Gamma$ may change when $L_{g}$ runs over $V_{m}\left(h^{0}\right)$ but at least we can fix the number of intersection points of $L_{g}$ and $\Gamma$ counting with multiplicity.

Lemma 4.6 Let $\left\{\Gamma_{p}\right\}$ be a family of curves in $\mathbb{C}^{2}$ with parameter $p \in P$ and let $d_{0}$ be natural so that $d_{0}>m+k$ where $k=\operatorname{dim} P$ and $m>0$. Then for generic $h^{0} \in G_{d_{0}}$ and every $p \in P$ the intersection number $L_{g} \cdot \Gamma_{p}$ is finite and it does not depend on $g \in G_{m}$. In particular, $L_{g}$ is not a component of $\Gamma_{p}$ for every $p$ and $g$.

Proof. Let $N$ be the maximal possible degree of the polynomial $\varphi(x)=f(x, h(x))$ where $h$ runs over $G_{d_{0}}$. Let $L$ be the curve $y=h(x)$. Suppose that $L \cdot \Gamma=N-l$. Then the degree of $f(x, h(x))=\sum_{i=0}^{N} a_{i} x^{i}$ is $N-l$. Note that the coefficients $a_{i}=0$ for $i>N-l$ only when $h$ belongs to an algebraic subvariety $\mathcal{A}(l)$ of $G_{d_{0}}$ which depends algebraically on $f$. If the leading coefficient of $\varphi$ does not depend on $h$ this subvariety is empty. Otherwise its codimension in $G_{d_{0}}$ is $l$ when $l<d_{0}$. Replace $\Gamma, f, \mathcal{A}(l)$ by $\Gamma_{p}, f_{p}, \mathcal{A}_{p}(l)$ and consider $\mathcal{B}(l)=\bigcup_{p \in P} \mathcal{A}_{p}$. Then $\mathcal{B}(l)$ is a closed algebraic subvariety in $G_{d_{0}}$ whose codimension is at least $l-k$. In particular, a generic polynomial $h^{0}$ from $G_{d_{0}}$ does not belong to $\mathcal{B}(k+1)$. Let $h, \tilde{h} \in G_{d_{0}}$ be such that $h$ has the same $k+1$ leading coefficients as $\tilde{h}$ does. Note that for every $p \in P$ and $l \leq k$ we have $h \in \mathcal{A}(l)$ iff $\tilde{h} \in \mathcal{A}(l)$. Hence for every $p \in P$ and $l \leq k$ if $h^{0} \in \mathcal{A}_{p}(l)-\mathcal{B}(k+1)$ then $h^{0}+g \in \mathcal{A}_{p}(l)-\mathcal{B}(k+1)$ for every $g \in G_{m}$ which is the desired conclusion. QED.

Thus suppose further that the function $L_{g} \cdot \Gamma$ is constant on $G_{m}$, i.e the points from $L_{g} \cap \Gamma$ do not go to infinity when $g$ runs over $G_{m}$. Note also that if $g$ is a small perturbation of $g^{0}$ in $G_{m}$ and $L_{g^{0}}$ meets $\Gamma$ normally at some point then $L_{g}$ meets $\Gamma$ normally at a nearby point. Therefore, in order to construct a subspace $V$ as in Lemma 4.5 we should take care of the set $\bar{w}=\left\{w_{1}, \ldots, w_{l}\right\}$ at which $\Gamma$ meets $L_{g^{0}}$ non-normally. Let $k_{i}$ be the local intersection number of $\Gamma$ and $L_{g^{0}}$ at $w_{i}$ and let 
$k=k_{1}+\ldots+k_{l}$.

Definition 4.7 We call $k$ the defect of $L_{g^{0}}$ relative to $\Gamma$.

Let $\mu_{w}$ be the multiplicity of $\Gamma$ at a point $w \in \Gamma$. Put $\mu(\Gamma)=\max \left(\mu_{w} \mid w \in \Gamma\right)$ and $\alpha(\Gamma):=\max (4 \log \mu(\Gamma), 1)$.

Proposition 4.8 Let $\alpha:=\alpha(\Gamma)$, and let $S_{k}(\Gamma)$ be the subset of $G_{m}$ such that for each $g \in S_{k}(\Gamma)$ the defect of $L_{g}$ relative to $\Gamma$ is at least $k$. Suppose that $k \leq m$. Then the following are true.

(i) $S_{k}(\Gamma)$ is an algebraic subvariety of $G_{m}$ which depends algebraically on $f$.

(ii) The codimension of $S_{k}(\Gamma)$ in $G_{m}$ is more than $(\log k) / \alpha$.

(iii) For every $g^{0} \notin S_{k}(\Gamma)$ there exists a nonzero polynomial $\omega$ in one variable such that $\operatorname{deg} \omega<m$ and every generic $\mathcal{C}^{\prime}$-curve $L_{g}$ from $V$ described in Remark 4.4 meets $\Gamma$ at the same number of points as $L_{g^{0}}$. Furthermore, if $k<m+1$ one can suppose that $\omega(0)=0$.

(iv) if $\Gamma$ does not contain lines parallel to the $y$-axis then $L_{g}$ is $\Gamma$ compatible for every $g \notin S_{k}(\Gamma)$.

Proof. Consider $L^{h}=\{y+h(x)=0\}$ for every $h \in G_{d_{0}}$. Let $w^{0} \in \Gamma$ and let $1<k^{0}<d_{0}$. We denote by $T_{0}\left(w^{0}, k^{0}\right)$ the subset of $G_{d_{0}}$ such that for every $h$ from this subset the local intersection number of $L^{h}$ and $\Gamma$ at $w^{0}$ is at least $k^{0}$. Our first aim is to estimate the codimension of $T_{0}\left(w^{0}, k^{0}\right)$ in $G_{d_{0}}$. Let $x^{0}$ be the $x$-coordinate of $w^{0}$ and let $\varphi(x, h)=f(x,-h(x))$. We denote by $\varphi_{s}$ the $s$-th derivative of $\varphi$ with respect to $x$. Note that $h \in T_{0}\left(w^{0}, k^{0}\right)$ iff

$$
\begin{gathered}
\left(x^{0}, h\left(x^{0}\right)\right)=w^{0} \\
\varphi_{s}\left(x^{0}, h\right)=0
\end{gathered}
$$

where $s=1, \ldots, k^{0}-1$. Let $\mu_{0}$ be the multiplicity of $\Gamma$ at $w^{0}$. Then the equations (4.1.s) holds automatically for $1 \leq s \leq \mu_{0}-1$. Let $h(x)=\sum_{i} c_{i} x^{i}$. One can rewrite equations (4.1) in the form of polynomial equations on the coefficients $\left\{c_{i}\right\}$

$$
\begin{gathered}
\Phi_{0}\left(c_{0}\right)=0, \\
\Phi_{s}\left(\left\{c_{i}\right\}\right)=0
\end{gathered}
$$

where $s=\mu_{0}, \ldots, k^{0}-1$. Without loss of generality we can suppose that $x^{0}=0$. Then (4.2.0.) means that $c_{0}$ coincides with the $y$-coordinate of $w^{0}$, and $\Phi_{s}$ is just the coefficient before the monomial $x^{s}$ in the polynomial $f(x,-h(x))$. Replacing $y$ by $y-c_{0}$ we can suppose that $w^{0}$ is the origin. Then $c_{0}=0$ and one can see that the codimension of $T_{0}\left(w^{0}, k^{0}\right)$ in $G_{d_{0}}$ is one more than the codimension of the affine algebraic variety given by the equations (4.2.s) which do not contain now the variable $c_{0}$. Consider three cases. 
Case 1: $k^{0}=\mu_{0} \geq 2$. The codimension of $T_{0}\left(w^{0}, k^{0}\right)$ in $G_{d_{0}}$ is $1>\left(\log k^{0}\right) / \alpha$.

Case 2: $k^{0}>\mu_{0} \geq 2$. The polynomial $f$ does not contain monomials of degree less then $\mu_{0}$. Furthermore, $(x)^{\mu_{0}}$ cannot be the only monomial of degree $\mu_{0}$ in $f$ with a nonzero coefficient since $k^{0}>\mu_{0}$. Hence $f$ contains monomials of type $y^{r} x^{\mu_{0}-r}$ (where $r>0$ ) with nonzero coefficients. Suppose also that $r_{0}$ is the maximum among such $r$ 's. Consider in (4.2) the equations with $s=\mu_{0} t$ for some natural $t$. The assumption on the monomials of degree $\mu_{0}$ in $f$ implies that $\Phi_{s}=\lambda\left(c_{t}\right)^{r_{0}}+\Psi_{s}$ where $\lambda$ is a nonzero constant, the degree of the polynomial $\Psi_{s}$ in variable $c_{t}$ is less than $r_{0}$. Denote by $E_{n}$ the equation (4.2.s) with $s=\left(\mu_{0}\right)^{n}$ where $n$ can be any number from 1 to $\left[\log \left(k^{0}-1\right) / \log \mu_{0}\right]([a]$ is the entire part of $a)$. We saw already that $E_{n}$ depends on $c_{t}$ with $t=\left(\mu_{0}\right)^{n-1}$. On the other hand $E_{n}$ does not depend on $c_{j}$ where $j=\left(\mu_{0}\right)^{i}$ and $i \geq n$. Hence the codimension of $T_{0}\left(w^{0}, k^{0}\right)$ in $G_{d_{0}}$ is $\left[\left(\log \left(k^{0}-1\right)\right) / \log \mu_{0}\right]>\left(\log \left(k^{0}-1\right)\right) /\left(2 \log \mu_{0}\right)>\left(\log k^{0}\right) /\left(4 \log \mu_{0}\right) \geq\left(\log k^{0}\right) / \alpha$.

Case 3: $\mu_{0}=1$. One can check that for every $s$ the equation (4.2.s) does not contain $c_{i}$ when $i>s$ and it contains only a linear term with $c_{s}$. Hence the codimension of $T_{0}\left(w^{0}, k^{0}\right)$ in $G_{d_{0}}$ is $k^{0}$. That is, it is at least $1+\left(\log k^{0}\right) / \alpha$ since $k^{0} \geq 2$.

Let $G$ be the subset of $G_{d_{0}}$ that consists of all $h$ of form $h=h^{0}+g$ where $h^{0}$ is fixed and $g$ runs over $G_{m}$. Let $T^{0}\left(w^{0}, k^{0}\right)=T_{0}\left(w^{0}, k^{0}\right) \cap G$. We need to find the codimension of $T^{0}\left(w^{0}, k^{0}\right)$ in $G$ in the case when $k^{0}<m$. Suppose that $g(x)=\sum_{i} b_{i} x^{i}$. Note that there is one-to one correspondence between coefficients $\left\{c_{i}\right\}$ and $\left\{b_{i}\right\}$ where $i=0, \ldots, m$. Since $\Phi_{s}$ does not contain unknowns $c_{i}$ with $i>k^{0}$ we see that the codimension of $T^{0}\left(w^{0}, k^{0}\right)$ in $G$ is at least $\left(\log k^{0}\right) / \alpha$ for singular $w^{0}$ and it is at least $1+\left(\log k^{0}\right) / \alpha$ when $w^{0}$ is regular.

Let $\bar{w}=\left(w_{1}, \ldots, w_{l}\right)$ be different points on $\Gamma$ and let $\bar{k}=\left(k_{1}, \ldots, k_{l}\right)$ where each $k_{i} \geq 2$. Consider $R_{0}(\bar{w}, \bar{k})=\bigcap_{i=1}^{l} T^{0}\left(w_{i}, k_{i}\right)$. Let $\hat{w}=\left(w_{1}, \ldots, w_{n}\right)$ where $n \leq l$. Suppose that all coordinates of $\hat{w}$ are different singular points on $\Gamma$. Put $R^{0}(\hat{w}, \bar{k})=\bigcup_{\bar{w}} R_{0}(\bar{w}, \bar{k})$ where the first $n$ coordinates of $\bar{w}$ are fixed and coincide with $\hat{w}$, and the last $l-n$ coordinates run over all $(l-n)$-tuples of different regular points on $\Gamma$. By construction, $R_{0}(\bar{w}, \bar{k})$ depends algebraically on $\bar{w}$ and $f$. Hence $R^{0}(\hat{w}, \bar{k})$ depends algebraically on $f$. Since $S_{k}(\Gamma)$ is a union of a finite number of sets of type $R^{0}(\hat{w}, \bar{k})$ (with $\left.k=k_{1}+\ldots+k_{l}\right)$ this yields (i).

Let $x(i)$ be the $x$-coordinate of $w_{i}$. Then the equations (4.2) for $w^{0}=w_{i}$ can be viewed as some equations on the $\left(k_{i}-1\right)$-jet of $g$ at $x(i)$ and they do not impose any restrictions on higher derivatives of $g$. The existence of Lagrange polynomials implies that when $k \leq m$ the codimension of the $R_{0}(\bar{w}, \bar{k})$ in $G$ is at least $\sum_{i}\left(\log k_{i}\right) / \alpha+(l-n)$. Since $k_{i} \geq 2$ we have $k_{1}+\ldots+k_{l} \leq k_{1} \cdots k_{l}$. Hence the codimension of $R^{0}(\hat{w}, \bar{k})$ in $G$ is at least $(\log k) / \alpha$ which is $(i i)$.

Let $g^{0} \notin S_{k}(\Gamma)$. Suppose that $L_{g^{0}}$ meets $\Gamma$ non-normally at the points from $\bar{w}$ only. By the Lagrange theorem, there exists a nonzero polynomial $\omega$ of degree $k-l$ such that it has zero of order $k_{i}-1$ at each point $x(i)$. Furthermore, if we allow the 
degree of $\omega$ to be $k-l+1$ then we can also suppose that that $\omega(0)=0$. Consider $g:=g^{0}+\tilde{g} \omega \in G_{m}$. Note that for generic $\tilde{g}$ the curve $L_{g}$ meets $\Gamma$ non-normally at the points of $\bar{w}$ only. In particular, it meets $\Gamma$ at the same number of points as $L_{g^{0}}$ does which is (iii). (Recall that due to Lemma 4.6 we suppose that the intersection numbers $L_{g} \cdot \Gamma$ and $L_{g^{0}} \cdot \Gamma$ are the same.) Now (iv) follows from Lemma 4.5. QED.

Corollary 4.9 Let $\hat{f}$ be a polynomial on $\mathbb{C}^{3}$. For every $g \in G_{m}$ consider the curve $\Gamma(g)$ that is the zero locus of the polynomial $f^{0}(x, y)=\hat{f}(x, y, g(x))$. Let $f(x, y)=\hat{f}\left(x, y,-y-h^{0}(x)\right)$ where $h^{0}$ is as before Lemma 4.3. Suppose that $\Gamma$ is the zero locus of $f$ and $S_{k}(\Gamma)$ is the same as in Proposition 4.8. If $k<m$ then

(i) for every $g^{0} \notin S_{k}(\Gamma)$ there exists a nonzero polynomial $\omega$ in one variable such that deg $\omega<m$ and every generic $\mathcal{C}_{\text {-curve }} L_{g}$ from $V$ described in Remark 4.4 meets $\Gamma\left(g^{0}\right)$ at the same number of points as $L_{g^{0}}$;

(ii) if $\Gamma\left(g^{0}\right)$ does not contain lines parallel to the $y$-axis then $L_{g}$ is $\Gamma\left(g^{0}\right)$ compatible for every $g \notin S_{k}(\Gamma)$.

Proof. Put $\psi(x)=f(x,-h(x))$ where $h(x)=h^{0}(x)+g^{0}(x)$. The roots of $\psi$ are the $x$-coordinates of the points where $L_{g^{0}}$ meets $\Gamma$. The points where $L_{g^{0}}$ meets $\Gamma$ non-normally correspond to the multiple roots of $\psi$ and the local intersection number of $L_{g^{0}}$ and $\Gamma$ at each of these points coincides with the corresponding multiplicity. Note that $f^{0}(x, y)=f(x, y)+\tilde{f}(x, y)$ where $\tilde{f}$ belongs to the ideal generated by the polynomial $y+h(x)$. Since $y+h(x) \equiv 0$ on $L_{g}$ we have $\psi(x)=f^{0}(x,-h(x))$. This implies that $L_{g^{0}}$ meets $\Gamma\left(g^{0}\right)$ at the same points as $L_{g^{0}}$ meets $\Gamma$ and with the same multiplicity. In particular, the defects of $L_{g^{0}}$ relative to $\Gamma$ and relative to $\Gamma\left(g^{0}\right)$ coincide. Let $x(i)$ be the $i$-th multiple root of $\psi$ where $i=1, \ldots, l$ and let $k_{i}$ be the multiplicity of this root. Suppose that $w_{i} \in \mathbb{C}^{2}$ is the point with $x$-coordinate $x(i)$ where $L_{g^{0}}$ meets $\Gamma\left(g^{0}\right)$. As we showed in the proof of Proposition $4.8 L_{g}$ meets $\Gamma\left(g^{0}\right)$ at $w_{i}$ with local intersection number at least $k_{i}$ when $g$ has the same $\left(k_{i}-1\right)$-jet at $x(i)$ as $g^{0}$ has. Since $k=k_{1}+\ldots+k_{l}<m$ there exists a nonzero polynomial $\omega(x)$ of degree less than $m$ which has zeros at $x(i)$ of multiplicity $k_{i}$ for every $i$. Consider generic $g(x)=g^{0}(x)+\omega(x) \tilde{g}(x)=0$ where $\tilde{g} \in G_{n}$ and $n=m-\operatorname{deg} \omega$. By Lemma 4.6, the intersection number $L_{g} \cdot \Gamma\left(g^{0}\right)$ does not depend on $g$, and since $g$ is generic the number of points where $L_{g}$ meets $\Gamma\left(g^{0}\right)$ normally is at least the same as the number of points where $L_{g^{0}}$ meets $\Gamma\left(g^{0}\right)$ normally. This implies (i). Lemma 4.5 yields (ii). QED.

Corollary 4.10 Let $\left\{\hat{f}_{p} \mid p \in P\right\}$ be a family of polynomials on $\mathbb{C}^{3}$. For every $g \in G_{m}$ consider the curve $\Gamma_{p}(g)$ that is the zero locus of the polynomial $f_{p}^{0}(x, y)=$ $\hat{f}_{p}(x, y, g(x))$. Let $f_{p}(x, y)=\hat{f}_{p}\left(x, y,-y-h^{0}(x)\right)$ where $h^{0}$ is as before Lemma 4.3 . Let $\Gamma_{p}$ be the zero locus of $f_{p}$. Suppose that $\theta=\max _{p} \alpha\left(\Gamma_{p}\right)$ where $\alpha\left(\Gamma_{p}\right)$ is as before 
Proposition 4.8. Suppose that $m>\exp (\theta \operatorname{dim} P)$. Then

(i) the closure $S(P)$ of $\bigcup_{p \in P} S_{m}\left(\Gamma_{p}\right)$ in $G_{m}$ is a proper algebraic subvariety where $S_{m}\left(\Gamma_{p}\right)$ has the same meaning as in Proposition 4.8;

(ii) for every $g^{0} \notin S(P)$ and every $p \in P$ there exists a nonzero polynomial $\omega_{p}$ in one variable such that for a generic element $g \in G_{m}$ of form $g=g^{0}+\tilde{g} \omega_{p}$ the curve $L_{g}$ meets $\Gamma_{p}\left(g^{0}\right)$ at the same number of points as $L_{g^{0}}$ does;

(iii) if $g^{0} \notin S(P)$ and none of the curves $\left\{\Gamma_{p}\left(g^{0}\right)\right\}$ contains lines parallel to the $y$-axis then $L_{g^{0}}$ is compatible relative to the family $\left\{\Gamma_{p}\left(g^{0}\right)\right\}$.

Proof. The codimension of $S(P)$ in $G_{m}$ is more than $(\log m) / \theta-\operatorname{dim} P>0$, by Proposition 4.8. Hence $G_{m}-S(P)$ is Zariski open which is the first statement of this Corollary. The second and the third statements follow from Corallary 4.9. QED.

\section{Main result.}

We shall consider first the three-dimensional case.

Proposition 5.1. Let $\left\{H_{p}\right\}$ be a family of hypersurfaces in $\mathbb{C}^{3}$ with parameter $p \in P$. Then there exists a coordinate system in $\mathbb{C}^{3}$ such that some plane in this system is strictly compatible relative to $\left\{H_{p}\right\}$.

Proof. Let $(x, y, z)$ be a coordinate system in $\mathbb{C}^{3}$ such that none of the surfaces $H_{p}$ contains a plane $z=$ const (it is enough to require that the restriction of the projection $(x, y, z) \rightarrow(x, z)$ to every $H_{p}$ is finite which can be done by Lemma 4.1). Let $\varphi$ be a polynomial in one variables such that $\operatorname{deg} \varphi>>\max _{p}\left(\operatorname{deg} f_{p}, \operatorname{dim} P\right)$. Put $q=(p, \varphi)$ and $f^{q}(x, y, z)=f_{p}(x+\varphi(z), y, z)$ where $f_{p}$ is a defining polynomial for $H_{p}$. Suppose that $Q$ is the variety of all $q$ 's and $H^{q}$ is the zero locus of $f^{q}$. Then $\left\{H^{q} \mid q \in Q\right\}$ is a family of hypersurfaces which is invariant under automorphisms of form $(x, y, z) \rightarrow(x+\varphi(z), y, z)$. If $Q_{0}$ is a subvariety of $Q$ that consists of $q=\left(p, \varphi_{0}\right)$ for some fixed $\varphi_{0}$ then the subfamily $\left\{H^{q} \mid q \in Q_{0}\right\}$ coincides with $\left\{H_{p} \mid p \in P\right\}$ after a polynomial coordinate substitution.

Let $h$ be a polynomial in one variable such that $\operatorname{deg} h>>\operatorname{deg} \varphi$. Replace $(x, y, z)$ by $(x, y+h(x), z)$. Then the equation of $H^{q}$ becomes $f^{q}(x, y+h(x), z)=f_{p}(x+$ $\varphi(z), y+h(x+\varphi(z)), z)=0$. By Lemma 4.1 the restriction of the projection $\rho$ : $\mathbb{C}^{3} \rightarrow \mathbb{C}^{2}$ (given by $\left.(x, y, z) \rightarrow(x, y)\right)$ to every $H^{q}$ is finite. When $h$ is fixed the image of the ramification set of this restriction is a curve $\Gamma^{q}$ in the $(x, y)$-plane which depends on $q \in Q$. If we replace $h$ by $h+g$ then each curve $\Gamma^{q}$ in the $(x, y)$-plane must be replaced by its image under automorphism $(x, y) \rightarrow(x, y+g(x))$. Suppose that $\operatorname{deg} g<<\operatorname{deg} h$ but still $\operatorname{deg} g>>\operatorname{dim} Q$. By Lemma 4.6 one can suppose that for every $q$ the intersection number of $\Gamma^{q}$ and the curve $L_{g}$ given by $y+g(x)=0$ is finite and does not depend on $g$. In particular, $L_{g}$ is not a component of $\Gamma^{q}$. Hence 
condition (2) from Definition 3.1 is true for $(x, z)$-plane $A_{0}$ with respect to the family of surfaces $\left\{H^{q} \mid q \in Q\right\}$.

Consider the family of curves $\Theta_{q}$ in $A_{0}$ which are the intersections $H^{q} \cap A_{0}$. That is, the equation of $\Theta_{q}$ is $f_{p}(x+\varphi(z), h(x+\varphi(z)), z)=0$. Show that none of these curves contain a line $z=c$. Consider the curves $\left\{\Lambda_{c, q}\right\}$ in the $(x, y)$-plane given by the equations $f_{p}(x+\varphi(c), y+h(x+\varphi(c)), c)=0$. Note that $\Theta_{q}$ contains the line $z=c$ iff $\Lambda_{c, q}$ contains the $y$-axis. But $\Lambda_{c, q}$ cannot contain the $y$-axis since the restriction of the projection $(x, y) \rightarrow y$ to every $\Lambda_{c, q}$ is finite by Lemma 4.1.

If $\Sigma$ is a curve in $\mathbb{C}^{2}$ denote by $\mu(\Sigma)$ the maximum of multiplicities of its points. Consider the curve $\Sigma$ in $A_{0}$ given by the equation $f_{p}(x, h(x), z)=0$. Note that $\mu(\Sigma)$ is at most $\operatorname{deg}_{z} f_{p}(x, y, z) \leq \operatorname{deg} f_{p}$ since this is the number of points at which a generic line $x=$ const meets $\Sigma$. Since $\Theta_{q}$ can be obtained from $\Sigma$ by an automorphism we see that $\max _{q \in Q} \mu\left(\Theta_{q}\right)$ is bounded by $\max _{p} \operatorname{deg} f_{p}<<\operatorname{deg} \varphi$. Hence $\max _{q \in Q} \alpha\left(\Theta_{q}\right)<<$ $\operatorname{deg} \varphi$ where $\alpha$ is the same as before Proposition 4.8. Corollary 4.10 implies that one can choose $\varphi=\varphi_{0}$ so that the $z$-axis in $A_{0}$ is $\Theta_{q}$ compatible for every $q$ of form $\left(p, \varphi_{0}\right)$ where $p$ is arbitrary. Hence condition (1) from Definition 3.1 is true for $A_{0}$ with respect to the subfamily $\left\{H^{q} \mid q \in Q_{0}\right\}$.

Let $\Gamma$ be the image in the $(x, y)$-plane of the ramification set of the restriction of $\rho$ to the surface given by $f^{q}(x, y, z)=0$. Note that $\Gamma^{q}$ can be obtained from $\Gamma$ by an automorphism of the $(x, y)$-plane and, therefore, $\alpha\left(\Gamma_{q}\right)=\alpha(\Gamma)$. Hence $M=\max _{q \in Q} \alpha\left(\Gamma^{q}\right)$ is independent from $h$. Since we have no restrictions on the degree of $h$ we can suppose from the beginning that $\operatorname{deg} h$ is much greater than $\exp (M \operatorname{dim} Q)$. Let $\operatorname{deg} g<\operatorname{deg} h$ but still $\operatorname{deg} g>>\max \left(\operatorname{deg} \varphi_{0}, \exp (M, \operatorname{dim} Q)\right)$. By virtue of Proposition 4.8 one can suppose that the following are true: for every $q \in Q$ there exists a nonzero polynomial $g$ such that $g(0)=0$ and the line $L_{0}$ (i.e., the $x$-axis) meets $\Gamma^{q}$ at the same number of points (counting without multiplicity) as the curve $L_{c g}$ for generic $c \in \mathbb{C}$. This gives conditions (3) from Definition 3.1 for $A_{0}$ with respect to the family $\left\{H^{q} \mid q \in Q\right\}$. That is, $A_{0}$ is strictly compatible relative to the family $\left\{H^{q} \mid q \in Q_{0}\right\}$ by Theorem 3.2.

QED.

Remark 5.2 Using the fact that $\operatorname{deg}_{z} f_{p}(x+\varphi(z), h(x+\varphi(z)), z)=\operatorname{deg}_{z} f_{p}(x+$ $\varphi(z), y+h(x+\varphi(z)), z)$, it is not difficult to check that every plane from a Zariski open neighborhood of $A_{0}$ in the variety of planes (i.e., every generic plane) is strictly compatible with respect to the subfamily $\left\{H^{q} \mid q \in Q_{0}\right\}$. In fact, one can construct more sophisticated coordinate substitutions such that every plane becomes strictly $H_{p}$ compatible for every $p \in P$. The same remark is applicable in the case of an arbitrary dimension.

Lemma 5.3 Let $H$ be a hypersurface in $\mathbb{C}^{n+1}$, let $\lambda$ be a coordinate function on $\mathbb{C}^{n+1}$, and let $R$ be a hyperplane in $\mathbb{C}^{n+1}$ which is not given by $\lambda=$ const. For $c \in \mathbb{C}^{\prime}$ 
put $C_{c}=\lambda^{-1}(c), \quad R_{c}=C_{c} \cap R, \quad H_{c}=H \cap C_{c}$. Suppose that for a generic $c \in \mathbb{C}$ the manifold $R_{c}$ is strictly $H_{c}$ compatible in $C_{c} \cong \mathbb{C}^{n}$ and that for every $c \in \mathbb{C}$ the set $R_{c}$ is not an irreducible component of $H_{c}$. Then $R$ is strictly $H$ compatible.

Proof. Let $S$ be a finite set in $\mathbb{C}$. Put $R(S)=R-\left(\lambda^{-1}(S) \cup H\right), C(S)=$ $\mathbb{C}^{n+1}-\left(\lambda^{-1}(S) \cup H\right), \quad \lambda_{S}=\left.\lambda\right|_{C(S)}$, and $\lambda_{S}^{\prime}=\left.\lambda\right|_{R(S)}$. Choose the finite set $S \subset \mathbb{C}$ so that the mappings $\lambda_{S}: C(S) \rightarrow \mathbb{C}^{\prime}-S$ and $\lambda_{S}^{\prime}: R(S) \rightarrow \mathbb{C}-S$ are fibrations. Let $F$ be the fiber of $\lambda_{S}$ and let $F^{\prime}$ be the fiber $\lambda_{S}^{\prime}$. Then, by assumption, the natural embedding $e: F^{\prime} \rightarrow F$ generates an isomorphism $e_{*}: \pi_{1}\left(F^{\prime}\right) \rightarrow \pi_{1}(F)$. We have also two other embeddings $i: F \rightarrow C(S)$ and $i^{\prime}: F^{\prime} \rightarrow R(S)$ which implies the commutative diagram

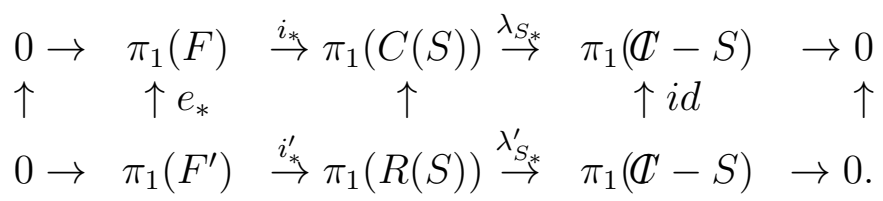

The five isomorphisms lemma implies that $\pi_{1}(C(S))$ and $\pi_{1}(R(S))$ are isomorphic. Since $R_{s}$ is not contained in $H_{s}$ for every $s$ one can choose simple loops $\gamma_{s}$ in $R(S)$ around each hypersurface $R_{s}$ with $s \in S$ such that $\gamma_{s}$ is contractible in $R-H$. Consider the natural embedding $j^{\prime}: R(S) \rightarrow R-H$. It generates an epimorphism $j_{*}^{\prime}: \pi_{1}(R(S)) \rightarrow \pi_{1}(R-H)$ and, obviously, $\left[\gamma_{s}\right] \in$ ker $j_{*}^{\prime}$ for every $s \in S$. Moreover, if $N^{\prime}$ is the smallest normal subgroup in $\pi_{1}(R(S))$ that contains all $\left[\gamma_{s}\right], s \in S$, then $\operatorname{ker} j_{*}^{\prime}=N^{\prime}$, which follows from two simple geometrical observations:

-each two-cell in $R-H$ becomes transversal to $\lambda^{-1}(S) \cap R$ after a perturbation, i.e. every contractible loop in $R-H$ can be viewed as a product of simple contractible loops around hyperplanes $R_{s}, s \in S$;

-every simple contractible loop of this type is conjugate to some $\left[\gamma_{s}\right]$ as an element of $\pi_{1}(R(S))$.

Similarly, we can consider the embedding $j: C(S) \rightarrow \mathbb{C}^{n}-H$. It generates an epimorphism $j_{*}: \pi_{1}(C(S)) \rightarrow \pi_{1}\left(\mathbb{C}^{n}-H\right)$. Then ker $j_{*}$ coincides with the smallest normal subgroup $N$ of $\pi_{1}(C(S))$ that contains all $\left[\gamma_{s}\right], s \in S$, where we treat $\left[\gamma_{s}\right]$ as an element of $\pi_{1}(C(S))$ now. This yields an isomorphism between $\pi_{1}(R-H)$ and $\pi_{1}\left(\mathbb{C}^{n}-H\right)$ which concludes the proof.

QED.

Theorem 5.4 Let $\left\{H_{p}\right\}$ be a family of hypersurfaces in $\mathbb{C}^{n}$ with parameter $p \in P$. Then there exists a polynomial coordinate system in $\mathbb{C}^{n+1}$ such that some plane is strictly $H_{p}$ compatible for every $p \in P$.

Proof. We shall use induction. The first step of induction is Proposition 5.1. 
Assume that for every family of hypersurfaces in $\mathbb{C}^{n}$ there exist a coordinate system such that some hyperplane in this system is strictly compatible relative to this family. Consider a family of hypersurfaces $\left\{H_{p}\right\}$ in $\mathbb{C}^{n+1}$. Let $\bar{x}=\left(x_{1}, \ldots, x_{n+1}\right)$ be a coordinate system in $\mathbb{C}^{n+1}$ and let $\lambda$ coincide with $x_{1}$ on $\mathbb{C}^{n+1}$. We can choose $\bar{x}$ so that none of $H_{p}$ contains a hyperplane $x_{1}=$ const since the restriction of the projection $\bar{x} \rightarrow\left(x_{1}, \ldots, x_{n}\right)$ to $H_{p}$ can be supposed to be finite, by the analogue of Lemma 4.1 in the case of higher dimensions. Put $C_{c}=\lambda^{-1}(c)$. We can view $H_{p, c}=H_{p} \cap C_{c}$ as a hypersurface in the fiber $C_{c}$. Put $Q=P \times \mathscr{C}$. Then we can consider $\left\{H_{p, c}=H^{q}\right\}$ with $q=(p, c) \in Q$ as a family of hypersurfaces in $\mathbb{C}^{n}$. By induction, a coordinate system $\bar{y}=\left(y_{2}, \ldots, y_{n+1}\right)$ in $\mathbb{C}^{n}$ can be chosed so that some hyperplane $E$ in $\mathbb{C}^{n}$ is strictly compatible relative to $\left\{H^{q}\right\}$. In particular, none of the hypersurfaces $H^{q}$ contain $E$. Let $R$ be the hyperplane $\tau^{-1}(E)$ in the coordinate system $\left(x_{1}, \bar{y}\right)$ in $\mathbb{C}^{n+1}$ where $\tau$ is the natural projection $\left(x_{1}, \bar{y}\right) \rightarrow \bar{y}$. By Lemma 5.3, the hyperplane $R$ is strictly compatible relative $\left\{H_{p}\right\}$. Therefore, we can reduce dimension by induction, which implies our Theorem.

QED.

\section{Bibliography.}

[1] O. Zariski, A theorem on the Poincaré group of an algebraic hypersurface, Annal of Math., 38(1937), 131-141.

[2] Sh. Kaliman, On the Jacobian conjecture, Proc. AMS, 117(1993), 45-51.

[3] E. van Kampen, On the connection between the fundamental groups of some related spaces, Amer. J. of Math., 55(1933), 261-267. 